\title{
Fatty acids and immune function: new insights into mechanisms
}

\author{
Parveen Yaqoob $^{1 *}$ and Philip C. Calder ${ }^{2}$ \\ ${ }^{1}$ Hugh Sinclair Unit of Human Nutrition, School of Food Biosciences, The University of Reading, Reading RG6 6AP, \\ United Kingdom \\ ${ }^{2}$ Institute of Human Nutrition, School of Medicine, University of Southampton, Southampton SO16 6YD, United Kingdom
}

Fatty acids are known to play diverse roles in immune cells. They are important as a source of energy, as structural components of cell membranes, as signaling molecules and as precursors for the synthesis of eicosanoids and similar mediators. Recent research has suggested that the localization and organisation of fatty acids into distinct cellular pools has a direct influence on the behaviour of a number of proteins involved in immune cell activation, including those associated with $\mathrm{T}$ cell responses, antigen presentation and fatty acid-derived inflammatory mediator production. This article reviews these studies and places them in the context of existing literature in the field. These studies indicate the existence of several novel mechanisms by which altered fatty acid availability can modulate immune responses and impact upon clinical outcomes.

Fatty acid: Fish oil: Omega-3: Eicosanoid: Lipid raft: T cell: Lymphocyte: Antigen presentation: Lipid body: Inflammation

Fatty acids are hydrocarbon chains of varying length with one end of the chain terminated by a methyl group and the other end by a reactive carboxyl group ${ }^{1}$. The hydrocarbon chain can be saturated, monounsaturated or polyunsaturated. Unsaturated fatty acids contain double bonds between pairs of adjacent carbon atoms; monounsaturated fatty acids contain one double bond, whereas polyunsaturated fatty acids (PUFAs) contain more than one double bond. There are only two classically essential fatty acids. These are linoleic and $\alpha$-linolenic acid, which cannot be synthesised de novo in animal cells and must therefore be obtained from the diet. Linoleic acid is an n-6 PUFA, described by its shorthand notation of $18: 2 n-6$, which refers to an 18-carbon fatty acid with two double bonds, the first of which is on carbon atom 6 from the methyl end. $\alpha$-Linolenic acid is an n-3 PUFA with a shorthand notation of $18: 3 n-3$, describing an 18-carbon fatty acid with three double bonds, the first being positioned at carbon atom 3 from the methyl end (Fig. 1). Dietary sources of linoleic and $\alpha$-linolenic acids include plant seeds and nuts, plant oils and margarines ${ }^{1}$. In most diets intake of linoleic acid is much greater (5 to 20-fold) than that of $\alpha$-linolenic acid ${ }^{2}$. Both essential fatty acids can be further elongated and desaturated in animal cells forming longer chain and more unsaturated members of the n-6 and n-3 families of PUFAs (Fig. 2). The metabolism of the $n-6$ and n-3 fatty acids is competitive, since both pathways employ the same set of enzymes. The major end-product of the $n-6$ pathway is arachidonic acid $(20: 4 n-6)$. This pathway is quantitatively the most important pathway of PUFA metabolism in humans, because linoleic acid is consumed in greater quantities than $\alpha$-linolenic acid. The major end-products of the n-3 pathway are eicosapentaenoic acid (EPA; 20:5n-3) and docosapentaenoic acid
(DPA; $22: 5 n-3)$; apparently relatively little $\alpha$-linolenic acid proceeds along the entire metabolic pathway to give rise to docosahexaenoic acid (DHA; $22: 6 n-3)^{2}$. Oily fish and fish oils contain a high proportion of the very long chain n-3 PUFAs, EPA, DPA and DHA ${ }^{3}$.

Fatty acids play diverse roles in all cells. They are important as a source of energy, as structural components of cell membranes so influencing the physical and functional properties of membranes, and as signaling molecules and regulators of gene expression ${ }^{1}$. In addition, some PUFAs including dihomo- $\gamma$-linolenic acid $(20: 3 n-6)$, arachidonic acid, EPA and DHA can serve as precursors for the synthesis of bioactive lipid mediators including prostaglandins, leukotrienes, lipoxins, and resolvins ${ }^{4-8}$. Within the immune system, different fatty acids act by differentially influencing cell membrane structure and function, cell signaling and gene expression, and patterns of lipid mediator production ${ }^{8-11}$. Through this variety of mechanisms fatty acids can modify the functional activities of cells of the immune system and the immune response as a whole. There are many reviews of this area ${ }^{8-19}$, and comprehensive coverage of all aspects is beyond the scope of this article. Instead this article will focus upon three specific areas in which significant new findings have been made in recent years. These are lipid rafts and T-cell signaling, antigen presentation and lipid body formation.

\section{Lipid rafts, $\mathbf{T}$ cell signaling and polyunsaturated fatty acids}

Lipid rafts are dynamic microenvironments in the exoplasmic leaflets of the phospholipid bilayer of plasma membranes. They are rich in saturated fatty acids, sphingolipids, cholesterol and glycosylphosphatidylinositol (GPI)-anchored

Abbreviations: DHA, docosahexaenoic acid; DPA, docosapentaenoic acid; EPA, eicosapentaenoic acid; GPI, glycosylphosphatidylinositol; LAT, linker of activated T cells; LT, leukotriene; MHC, major histocompatability complex; PG, prostaglandin; PUFA, polyunsaturated fatty acid; TCR, T-cell receptor.

* Corresponding author: Dr P. Yaqoob, fax +44 118931 0800, email P.Yaqoob@reading.ac.uk 
<smiles>CCCCC/C=C\C/C=C\CCCCCCCC(=O)O</smiles>

Linoleic acid $(18: 2 n-6)$

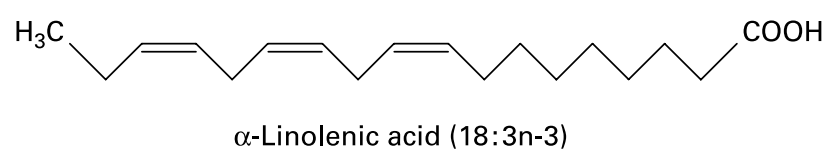

Fig. 1. The structure of the two essential fatty acids.

proteins ${ }^{20,21}$. Rafts serve as platforms to facilitate the association of signaling molecules and interactions and crosstalk between cell types ${ }^{20,21}$. Activation of the proteins within rafts by an extracellular ligand can result in rapid clustering, which appears to be important for signal transduction in both $\mathrm{T}$ and B lymphocytes ${ }^{22-25}$. The T-cell receptor (TCR) clusters within lipid rafts upon contact with an antigen presenting cell, forming an 'immunological synapse', or contact zone, where intracellular signaling is thought to be initiated. Interestingly, the role of membrane rafts in Th1 and Th2 cells appears to be markedly different, with TCR activation in Th1 cells being dependent on rafts, while that in Th2 cells is not ${ }^{26}$. The reason for this difference is not clear, but has been suggested to be due to differences in the composition, distribution or quantity of lipid rafts ${ }^{27}$.

Some key signaling proteins, such as the tyrosine kinase lck and the signaling molecule linker of activated T cells (LAT), are retained in $\mathrm{T}$ cell rafts via acylation, while other important signaling proteins, including CD4, ZAP70 and phospholipase C- $\gamma 1$, translocate to rafts upon stimulation of the TCR. Acylated proteins that are anchored to the inner lipid leaflet are displaced from rafts when $\mathrm{T}$ cells are treated with long chain n-3 PUFAs and to a somewhat lesser extent also with $n-6$ PUFAs $^{28}$. In contrast, GPI-anchored proteins remain located in detergent-resistant membranes with PUFA treatment $^{28}$. Thus, PUFAs appear to selectively alter the protein composition of the inner membrane lipid leaflet of $\mathrm{T}$ cells. Notably the extent of displacement of acylated proteins from detergent-resistant membranes correlates with impairment of calcium signaling indicating a functional impact of these alterations $^{28}$. PUFAs are readily esterified to the $s n-2$ position of membrane phospholipids of both rafts and the bulk plasma membrane thereby altering raft protein localization ${ }^{29}$. Feeding mice on an n-3 PUFA-rich diet resulted in incorporation of EPA and DHA into the lipids of the rafts of spleen $T$ cells and this was associated with a decreased sphingomyelin content ${ }^{30}$. Hence, incorporation of PUFAs into membrane lipids is a likely mechanism for protein displacement from rafts. Phosphorylation of LAT is the most upstream step that is inhibited
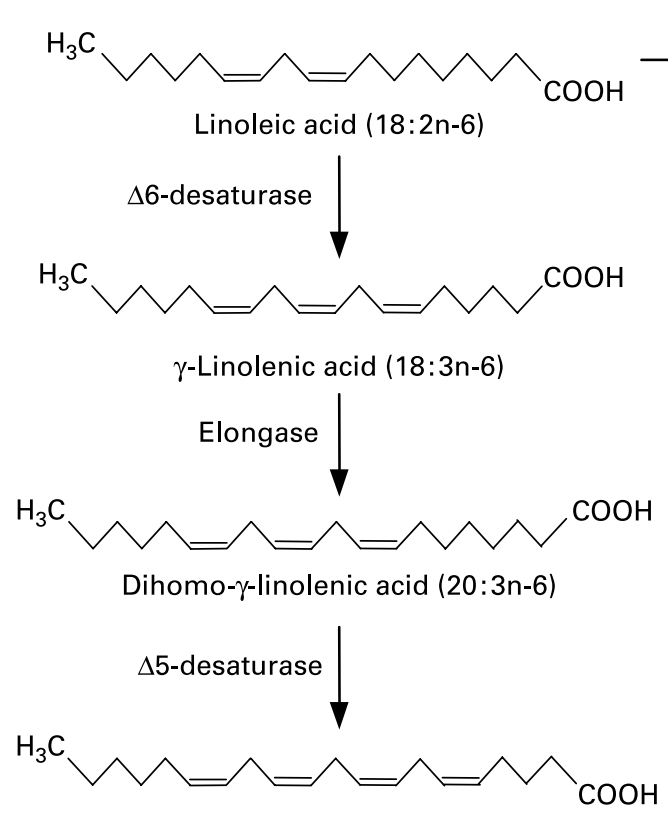

Arachidonic acid $(20: 4 n-6)$
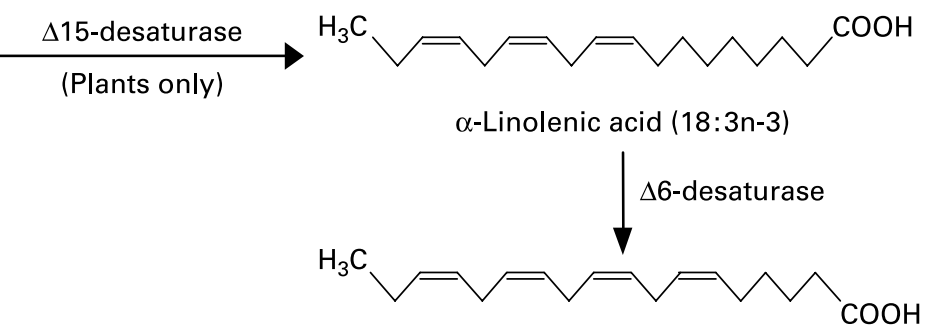

Stearidonic acid $(18: 4 n-3)$
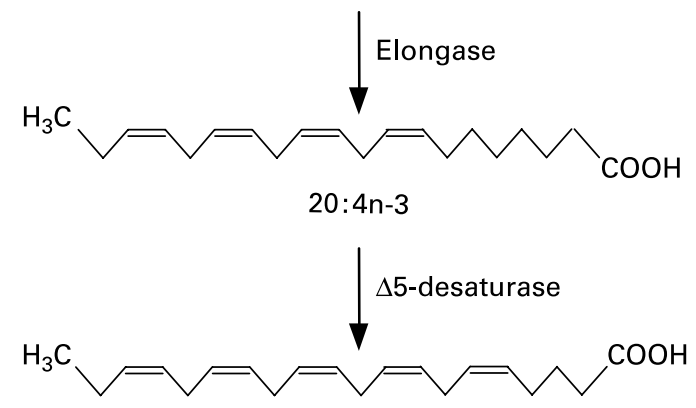

Eicosapentaenoic acid (EPA; 20:5n-3)

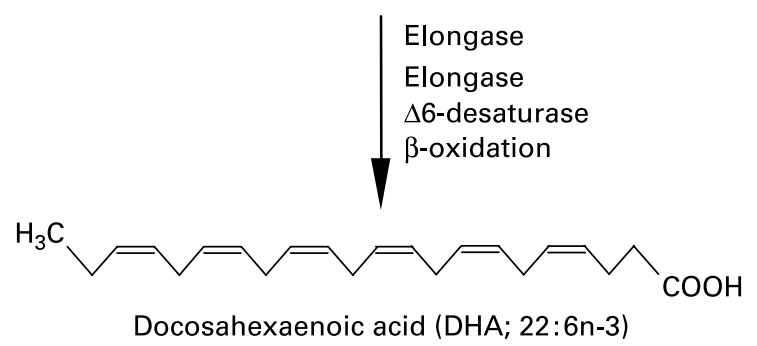

Fig. 2. The metabolic pathways by which essential fatty acids are converted to longer chain, more unsaturated derivatives. 
by long chain $\mathrm{n}-3$ PUFA treatment of $\mathrm{T}$ cells $\mathrm{s}^{31}$ and it appears that LAT displacement from rafts is a molecular mechanism mediating inhibition of $\mathrm{T}$ cell responses by $\mathrm{n}-3$ PUFAs, at least in vitro ${ }^{32}$. Importantly, animal studies have shown that dietary fish oil affects early signaling events in $\mathrm{T}$ cells such as phosphorylation of phospholipase $\mathrm{C}-\gamma 1^{33}$ and have linked alterations of $\mathrm{T}$ cell rafts by dietary $\mathrm{n}-3$ PUFAs with functional changes such as decreased proliferation and interleukin-2 production $^{34}$. There is no information regarding the extent to which lipid rafts in human lymphocytes could be modulated by dietary PUFAs, although human lymphocyte lipids are readily modified by fish oil supplementation ${ }^{35-38}$.

\section{Polyunsaturated fatty acids and antigen presentation}

Studies conducted in the early to mid $1990 \mathrm{~s}$ found that cell surface expression of major histocompatibility complex (MHC) II and antigen presentation via MHC II are decreased following in vitro exposure of antigen presenting cells to EPA or DHA ${ }^{39-43}$. There are limited studies investigating the effect of dietary PUFAs on MHC II expression ${ }^{44-46}$. The most thorough study of this type is that of Sanderson $e t a l{ }^{46}$ who showed that feeding a fish oil-rich diet to rats resulted in decreased expression of MHC II on dendritic cells which was associated with a decreased capacity to present antigen to antigen-sensitised spleen $T$ cells. The reduction in antigen presentation was probably much greater than could be explained by the reduction in MHC II expression, suggesting that other interactions between antigen presenting cells and T lymphocytes were affected by dietary n-3 PUFAs. Indeed, levels of CD2, CD11a and CD18 were also decreased on dendritic cells from fish oil-fed rats ${ }^{46}$.

Recently the effect of PUFAs on MHC I expression and on MHC I-mediated antigen presentation were reported for the first time ${ }^{47}$. Expression of MHC I was decreased with in vitro treatment of B lymphocytes with arachidonic acid or DHA. The effect was fatty acid concentration dependent, with arachidonic acid being slightly more effective than DHA. There was a functional effect of the reduced MHC I expression: cytotoxic $\mathrm{T}$ lymphocyte mediated lysis of target cells enriched with either arachidonic acid or DHA was decreased in a fatty acid concentration dependent manner. By blocking of resident MHC I molecules on the cell surface, it was identified that arachidonic acid and DHA decreased the surface appearance of new MHC I. Arachidonic acid and DHA were shown to decrease cell surface MHC I expression by slowing flow of new class I molecules from the endoplasmic reticulum to the Golgi. The lower forward trafficking rate could account for the lower level of MHC I surface expression. The finding of arachidonic acid- and DHA-induced inhibition of MHC I trafficking ${ }^{47}$ highlights a novel mechanism by which fatty acids affect the biology of antigen presentation. EPA was not examined in this work. Furthermore the work was restricted to in vitro investigations and there is an obvious need to examine these effects and mechanisms in a dietary setting.

\section{Lipid bodies as structures for generation of fatty acid-derived mediators}

Eicosanoids and other fatty acid-derived lipid mediators have numerous roles in the regulation of immune and inflammatory responses $^{4-7}$, the true extent of which has probably not yet been realised. It is becoming clear that the regulation of eicosanoid formation involves activation of enzymes at specific intracellular sites and that this local generation of eicosanoids might be facilitated by the presence of lipid bodies present within many (if not all) cell types. Lipid bodies within eosinophils increase in number following an inflammatory stimulus and appear to contain all of the enzymes necessary for eicosanoid synthesis ${ }^{48,49}$. Unlike lipid rafts, these distinct intracellular domains are not resistant to detergent solubilization and there are consequently some methodological limitations to their study. Since it is not yet possible to isolate lipid bodies, their structure and composition have not been elucidated.

Novel techniques have been used to cross-link newly-synthesized leukotriene (LT) $\mathrm{C}_{4}$ at sites of synthesis within eosinophils and to follow its fate upon stimulation ${ }^{48}$. This approach demonstrated that $\mathrm{LTC}_{4}$ formation does indeed occur in lipid bodies and that, depending on the nature of the stimulus, $\mathrm{LTC}_{4}$ can be either targeted towards the perinuclear membrane or released into the extracellular milieu ${ }^{48,50}$. Like lipid rafts, the distribution of lipid bodies can be polarized, but it is not clear whether those producing eicosanoids destined to be secreted are located close to the plasma membrane, while those that are perinuclear produce eicosanoids solely for autotropic effects. Recent studies have identified roles for lipid bodies in inflammatory mediator synthesis in a range of situations. A potential role for lipid bodies in sepsis is suggested by the observation that their numbers are higher in leukocytes from septic patients compared with healthy patients and that they are inducible by lipopolysaccharide in murine macrophages ${ }^{51}$. Administration of lipopolysaccharide to mice resulted in increased lipid body content of peritoneal cells and this was associated with increased prostaglandin $(\mathrm{PG}) \mathrm{E}_{2}$ and $\mathrm{LTB}_{4}$ in peritoneal lavage fluid ${ }^{52}$. Lipid body formation and presence of inflammatory mediators was examined in mice fed different oils prior to lipopolysaccharide exposure: olive oil was found to decrease peritoneal leukocyte infiltration, lipid body formation and $\mathrm{PGE}_{2}$ and $\mathrm{LTB}_{4}$ levels ${ }^{52}$. This was associated with a significant reduction in mortality ${ }^{52}$. Fish oil was not examined in that study but it might be expected to have similar effects since it is known that dietary fish oil decreases in vivo and ex vivo inflammatory mediator responses to lipopolysaccharide $^{53,54}$, and improves survival ${ }^{55,56}$. Other recent studies have attempted to address the mechanism of lipid body formation in the different contexts. In murine models of allergy, lung eosinophil influx and lipid body formation occurred in parallel in response to an allergic challenge ${ }^{57}$. 5-Lipoxygenase expression and $\mathrm{LTC}_{4}$ production were associated with lipid bodies. The induction of lipid body formation appeared to involve either eotaxin or RANTES acting via the CCR3 receptor ${ }^{57}$. Infection of macrophages with Mycobacterium bovis bacillus induced lipid body formation which was associated with increased $\mathrm{PGE}_{2}$ production and localization of cyclooxygenase- 2 to the lipid bodies ${ }^{58}$. The authors confirmed that lipid bodies were the main site of new $\mathrm{PGE}_{2}$ synthesis. The effect of infection on induction of lipid bodies was inhibited in toll-like receptor 2 , but not toll-like receptor 4 , deficient mice ${ }^{58}$, suggesting a role for the former in lipid body formation in macrophages in response to infection. It is clear that more needs to be understood about lipid bodies in the contexts of immune activation 
and inflammation in humans and of formation of different families of lipid mediators from different substrate fatty acids.

\section{Conclusion}

Recent studies have focused attention on the central role of fatty acids in immune cell regulation, highlighting that their location and organization within cellular lipids has a direct influence on the behaviour of a number of proteins involved in immune cell activation, including those associated with $\mathrm{T}$ cell responses, antigen presentation and fatty acid-derived inflammatory mediator production. Studies have tended to focus upon PUFAs per se or on PUFAs of different types (e.g. n-6 vs. n-3). While these studies clearly suggest that the fatty acid composition of lipid domains can strongly influence immune cell activation and functional responses, they have been mainly conducted in model systems and the relevance to the human setting requires urgent investigation. Nevertheless, the studies described herein indicate several novel mechanisms by which altered fatty acid availability can modulate immune responses and impact upon clinical outcomes relevant to infection and to inflammatory conditions.

\section{Conflict of interest statement}

PCC has research funding from B. Braun Melsungen, receives consultancy fees from Equazen Ltd. and Royal Dutch Numico and speaking fees from Solvay Healthcare, B. Braun Melsungen and Fresenius Kabi. PY has no conflicts of interest to declare. PCC and PY co-wrote the manuscript.

\section{References}

1. Calder PC \& Burdge GC (2004) Fatty acids. In Bioactive Lipids, pp. 1-36 [A Nicolaou and G Kokotos, editors]. Bridgewater: The Oily Press.

2. Burdge GC \& Calder PC (2006) Dietary $\alpha$-linolenic acid and health-related outcomes: a metabolic perspective. Nutrition Research Reviews 19, 26-52.

3. British Nutrition Foundation (1999) Briefing Paper: $n$-3 Fatty Acids and Health. London: British Nutrition Foundation.

4. Lewis RA, Austen KF \& Soberman RJ (1990) Leukotrienes and other products of the 5-lipoxygenase pathway: biochemistry and relation to pathobiology in human diseases. New England Journal of Medicine 323, 645-655.

5. Tilley SL, Coffman TM \& Koller BH (2001) Mixed messages: modulation of inflammation and immune responses by prostaglandins and thromboxanes. J Clin Invest 108, 15-23.

6. Vachier I, Chanez P, Bonnans C, Godard P, Bousquet J \& Chavis C (2002) Endogenous anti-inflammatory mediators from arachidonate in human neutrophils. Biochemical and Biophysical Research Communications 290, 219-224.

7. Serhan CN, Arita M, Hong S \& Gotlinger K (2004) Resolvins, docosatrienes, and neuroprotectins, novel omega-3-derived mediators, and their endogenous aspirin-triggered epimers. Lipids 39, 1125-1132.

8. Calder PC (2006) N-3 polyunsaturated fatty acids, inflammation, and inflammatory diseases. American Journal of Clinical Nutrition 83, 1505S-1519S.

9. Miles EA \& Calder PC (1998) Modulation of immune function by dietary fatty acids. Proceedings of the Nutrition Society $\mathbf{5 7}$, 277-292.
10. Calder PC (2003) N-3 Polyunsaturated fatty acids and inflammation: from molecular biology to the clinic. Lipids 38, $342-352$.

11. Yaqoob P (2003) Fatty acids as gatekeepers of immune cell regulation. Trends in Immunology 24, 639-645.

12. Calder PC (1996) Sir David Cuthbertson Medal Lecture: Immunomodulatory and anti-inflammatory effects of omega-3 polyunsaturated fatty acids. Proceedings of the Nutrition Society $\mathbf{5 5}$, $737-774$

13. Calder PC (1998) Dietary fatty acids and lymphocyte functions. Proceedings of the Nutrition Society 57, 487-502.

14. Calder PC (2001) Polyunsaturated fatty acids, inflammation and immunity. Lipids 36, 1007-1024.

15. Calder PC, Yaqoob P, Thies F, Wallace FA \& Miles EA (2002) Fatty acids and lymphocyte functions. British Journal of Nutrition 87, S31-S48.

16. Stulnig TM \& Zeyda M (2004) Immunomodulation by polyunsaturated fatty acids: impact on T-cell signalling. Lipids 39, $1171-1175$.

17. Hwang D (2000) Fatty acids and immune responses-a new perspective in searching for clues to mechanism. Annual Review of Nutrition 20, 431-456.

18. Sijben JWC \& Calder PC (2007) Differential immunomodulation with long-chain n-3 PUFA in health and disease. Proceedings of the Nutrition Society 66, 237-259.

19. Yaqoob P (2004) Fatty acids and the immune system: from basic science to clinical applications. Proceedings of the Nutrition Society 63, 89-104.

20. Pike LJ (2003) Lipid rafts: bringing order to chaos. Journal of Lipid Research 44, 655-667.

21. Simons K \& Toomre D (2000) Lipid rafts and signal transduction. Nature Reviews Molecular and Cell Biology 1, 31-39.

22. Pierce SK (2002) Lipid rafts and B cell activation. Nature Reviews Immunology 2, 96-105.

23. Katagiri YU, Kiyokawa N \& Fujimoto J (2001) A role for lipid rafts in immune cell signalling. Microbiology and Immunology 45, 1-8.

24. Razzaq TM, Ozegbe P, Jury EC, Sembi P, Blackwell NM \& Kabouridis PS (2004) Regulation of T-cell receptor signaling by membrane microdomains. Immunology 113, 413-426.

25. Harder $\mathrm{T}$ (2004) Lipid raft domains and protein networks in T-cell receptor signal transduction. Current Opinion in Immunology 16, 353-359.

26. Balamuth F, Leitenberg D, Unternaehrer J, Mellman I \& Bottomly K (2001) Distinct patterns of membrane microdomain partitioning in Th1 and Th2 cells. Immunity 15, 729-738.

27. Horejsi V (2003) The roles of membrane microdomains (rafts) in $\mathrm{T}$ cell activation. Immunology Reviews 191, 148-164.

28. Stulnig T, Berger M, Sigmund T, Raderstorff D, Stockinger H \& Waldhausl W (1998) Polyunsaturated fatty acids inhibit T cell signal transduction by modification of detergent-soluble membrane domains. Journal of Cell Biology 143, 637-644.

29. Stulnig TM, Huber J, Leitinger N, Imre E-M, Angelisoval P, Nowotny P \& Waldhausl W (2001) Polyunsaturated eicosapentaenoic acid displaces proteins from membrane rafts by altering raft lipid composition. Journal of Biological Chemistry 276, 37335-37340.

30. Fan YY, McMurray DN, Ly LH \& Chapkin RS (2003) Dietary n-3 polyunsaturated fatty acids remodel mouse T-cell lipid rafts. Journal of Nutrition 133, 1913-1920.

31. Zeyda M, Szekeres AB, Saemann MD, Geyregger R, Stockinger H, Zlabinger GJ, Waldhausl W \& Stulnig TM (2003) Suppression of $\mathrm{T}$ cell signaling by polyunsaturated fatty acids: selectivity in inhibition of mitogen-activated protein kinase and nuclear factor activation. Journal of Immunology 170, 6033-6039.

32. Zeyda M, Staffler G, Horejsi V \& Waldhausl W (2002) LAT displacement from lipid rafts as a molecular mechanism for 
the inhibition of $\mathrm{T}$ cell signalling by polyunsaturated fatty acids. Journal of Biological Chemistry 277, 28418-28423.

33. Sanderson P \& Calder PC (1998) Dietary fish oil appears to prevent the activation of phospholipase $\mathrm{C}-\gamma$ in lymphocytes. Biochimica et Biophysica Acta 1392, 300-308.

34. Fan YY, Ly LH, Barhoumi R, McMurray DN \& Chapkin RS (2004) Dietary docosahexaenoic acid suppresses T cell protein kinase $\mathrm{C} \theta$ lipid raft recruitment and IL-2 production. Journal of Immunology 173, 6151-6160.

35. Gibney MJ \& Hunter B (1993) The effects of short- and long-term supplementation with fish oil on the incorporation of $n-3$ polyunsaturated fatty acids into cells of the immune system in healthy volunteers. European Journal of Clinical Nutrition 47, 255-259.

36. Yaqoob P, Pala HS, Cortina-Borja M, Newsholme EA \& Calder PC (2000) Encapsulated fish oil enriched in $\alpha$-tocopherol alters plasma phospholipid and mononuclear cell fatty acid compositions but not mononuclear cell functions. European Journal of Clinical Investigation 30, 260-274.

37. Thies F, Nebe-von-Caron G, Powell JR, Yaqoob P, Newsholme EA \& Calder PC (2001) Dietary supplementation with $\gamma$-linolenic acid or fish oil decreases $\mathrm{T}$ lymphocyte proliferation in healthy older humans. Journal of Nutrition 131, 1918-1927.

38. Rees D, Miles EA, Banerjee T, Wells SJ, Roynette CE, Wahle KWJW \& Calder PC (2006) Dose-related effects of eicosapentaenoic acid on innate immune function in healthy humans: a comparison of young and older men. American Journal of Clinical Nutrition 83, 331-342.

39. Fujikawa M, Yamashita N, Yamazaki K, Sugiyama E, Suzuki H \& Hamazaki T (1992) Eicosapentaenoic acid inhibits antigenpresenting cell function of murine splenocytes. Immunology 75, 330-335.

40. Khair-el-Din TA, Sicher SC, Vazquez MA \& Lu CY (1996) Inhibition of macrophage nitric-oxide production and Ia-expression by docosahexaenoic acid, a constituent of fetal and neonatal serum. American Journal of Reproductive Immunology 36, 1-10.

41. Khair-el-Din TA, Sicher SC, Vazquez MA, Wright WJ \& Lu CY (1995) Docosahexaenoic acid, a major constituent of fetal serum and fish oil diets, inhibits IFN gamma-induced Ia-expression by murine macrophages in vitro. Journal of Immunology 154, 1296-1306.

42. Weatherill AR, Lee JY, Zhao L, Lemay DG, Youn HS \& Hwang DH (2005) Saturated and polyunsaturated fatty acids reciprocally modulate dendritic cell functions mediated through TLR4. Journal of Immunology 174, 5390-5397.

43. Hughes DA, Southon S \& Pinder AC (1996) (n-3) Polyunsaturated fatty acids modulate the expression of functionally associated molecules on human monocytes in vitro. Journal of Nutrition 126, 603-610.

44. Huang SC, Misfeldt ML \& Fritsche KL (1992) Dietary fat influences Ia antigen expression and immune cell populations in the murine peritoneum and spleen. Journal of Nutrition 122, 1219-1231.

45. Hughes DA, Pinder AC, Piper Z, Johnson IT \& Lund EK (1996) Fish oil supplementation inhibits the expression of major histocompatibility complex class II molecules and adhesion molecules on human monocytes. American Journal of Clinical Nutrition 63, 267-272.

46. Sanderson P, MacPherson GG, Jenkins CH \& Calder PC (1997) Dietary fish oil diminishes the antigen presentation activity of rat dendritic cells. Journal of Leukocyte Biology 62, 771-777.

47. Shaikh SR \& Edidin M (2007) Immunosuppressive effects of polyunsaturated fatty acids on antigen presentation by HLA class I molecules. Journal of Lipid Research 48, 127-138.

48. Bandeira-Melo C, Bozza PT \& Weller PF (2002) The cellular biology of eosinophil eicosanoid formation and function. Journal of Allergy and Clinical Immunology 109, 393-400.

49. Bozza PT, Melo RC \& Bandeira-Melo C (2007) Leukocyte lipid bodies regulation and function: contribution to allergy and host defense. Pharmacology and Therapeutics 113, 30-49.

50. Bandeira-Melo C, Phoofolo M \& Weller PF (2001) Extranuclear lipid bodies, elicited by CCR3-mediated signaling pathways, are the sites of chemokine-enhanced leukotriene $\mathrm{C}_{4}$ production in eosinophils and basophils. Journal of Biological Chemistry 276, 22779-22787.

51. Pacheco P, Bozza FA, Gomes RN, Bozza M, Weller PF, CastroFaria-Neto HC \& Bozza PT (2002) Lipopolysaccharide-induced leukocyte lipid body formation in vivo: innate immunity elicited intracellular loci involved in eicosanoid metabolism. Journal of Immunology 169, 6498-6506.

52. Leite MS, Pacheco P, Gomes RN, Guedes AT, Castro-FariaNeto HC, Bozza PT \& Koatz VL (2005) Mechanisms of increased survival after lipopolysaccharide-induced endotoxic shock in mice consuming olive oil-enriched diet. Shock 23, 173-178.

53. Yaqoob P \& Calder PC (1995) Effects of dietary lipid manipulation upon inflammatory mediator production by murine macrophages. Cellular Immunology 163, 120-128.

54. Sadeghi S, Wallace FA \& Calder PC (1999) Dietary lipids modify the cytokine response to bacterial lipopolysaccharide in mice. Immunology 96, 404-410.

55. Mascioli E, Leader L, Flores E, Trimbo S, Bistrian B \& Blackburn G (1988) Enhanced survival to endotoxin in guinea pigs fed IV fish oil emulsion. Lipids 23, 623-625.

56. Mascioli EA, Iwasa Y, Trimbo S, Leader L, Bistrian BR \& Blackburn GL (1989) Endotoxin challenge after menhaden oil diet: effects on survival of guinea pigs. American Journal of Clinical Nutrition 49, 277-282.

57. Vieira-de-Abreu A, Assis EF, Gomes GS, Castro-Faria-Neto HC, Weller PF, Bandeira-Melo C \& Bozza PT (2005) Allergic challenge-elicited lipid bodies compartmentalize in vivo leukotriene $\mathrm{C} 4$ synthesis within eosinophils. American Journal of Respiratory Cell and Molecular Biology 33, 254-261.

58. D'Avila H, Melo RC, Parreira GG, Werneck-Barroso E, CastroFaria-Neto HC \& Bozza PT (2006) Mycobacterium bovis bacillus Calmette-Guérin induces TLR2-mediated formation of lipid bodies: intracellular domains for eicosanoid synthesis in vivo. Journal of Immunology 176, 3087-3097. 